\title{
ZERO-NONZERO PATTERNS THAT ALLOW OR REQUIRE AN INERTIA SET RELATED TO DYNAMICAL SYSTEMS*
}

\author{
WEI $\mathrm{GAO}^{\dagger}$ AND YANLING SHAO $\ddagger$
}

\begin{abstract}
The inertia of an $n \times n$ real matrix $B$, denoted by $\mathrm{i}(B)$, is the ordered triple $\mathrm{i}(B)=\left(i_{+}(B), i_{-}(B), i_{0}(B)\right)$, in which $i_{+}(B), i_{-}(B)$ and $i_{0}(B)$ are the numbers of its eigenvalues (counting multiplicities) with positive, negative and zero real parts, respectively. The inertia of an $n \times n$ zero-nonzero pattern $\mathcal{A}$ is the set $\mathrm{i}(\mathcal{A})=\{\mathrm{i}(B) \mid B \in Q(\mathcal{A})\}$. For $n \geq 2$, let $\mathbb{S}_{n}^{*}=\{(0, n, 0),(0, n-1,1),(1, n-1,0),(n, 0,0),(n-1,0,1),(n-1,1,0)\}$. An $n \times n$ zero-nonzero pattern $\mathcal{A}$ allows $\mathbb{S}_{n}^{*}$ if $\mathbb{S}_{n}^{*} \subseteq \mathrm{i}(\mathcal{A})$ and requires $\mathbb{S}_{n}^{*}$ if $\mathbb{S}_{n}^{*}=\mathrm{i}(\mathcal{A})$. In this paper, it is shown that there are no zero-nonzero patterns for order $n \geq 2$ that require $\mathbb{S}_{n}^{*}$. Also, a complete characterization of zero-nonzero star patterns of order $n \geq 3$ that allow $\mathbb{S}_{n}^{*}$ is given.
\end{abstract}

Key words. Zero-nonzero pattern, Eigenvalues, Inertia, Digraph.

AMS subject classifications. 15B35, 05C20, 15A18, 05C50.

1. Introduction. An $n \times n$ zero-nonzero pattern is an $n \times n$ matrix $\mathcal{A}$ with entries from $\{*, 0\}$, where $*$ is nonzero. A real matrix $B=\left(b_{i j}\right)$ is a realization of $\mathcal{A}$ if $b_{i j} \neq 0$ if and only if the $(i, j)$-entry of $\mathcal{A}$ is $*$. The zero-nonzero pattern class $Q(\mathcal{A})$ is the set of all realizations of $\mathcal{A}$. Two zero-nonzero patterns are equivalent if one can be obtained from the other by any combination of transposition and permutation similarity. If we assign each nonzero entry of $\mathcal{A}$ a sign ( + or - ), then we can get a sign pattern $\mathcal{B}$, whose entries come from the set $\{+,-, 0\}$. The sign pattern $\mathcal{B}$ is called a signing of $\mathcal{A}$.

The inertia of an $n \times n$ real matrix $B$, denoted by $\mathrm{i}(B)$, is the ordered triple $\mathrm{i}(B)=\left(i_{+}(B), i_{-}(B), i_{0}(B)\right)$, in which $i_{+}(B), i_{-}(B)$ and $i_{0}(B)$ are the numbers of its eigenvalues (counting multiplicities) with positive, negative and zero real parts, respectively. The inertia of an $n \times n$ zero-nonzero pattern $\mathcal{A}$ is the set $\mathrm{i}(\mathcal{A})=$ $\{\mathrm{i}(B) \mid B \in Q(\mathcal{A})\}([11])$.

In [2], motivated by the possible onset of instability in dynamical systems associated with a zero eigenvalue, the inertia set $S_{n}$ with $n \geq 2$ is defined as

$$
\mathbb{S}_{n}=\{(0, n, 0),(0, n-1,1),(1, n-1,0)\} .
$$

For a zero-nonzero pattern $\mathcal{A},\left(i_{+}, i_{-}, i_{0}\right) \in \mathrm{i}(\mathcal{A})$ if and only if its reversal $\left(i_{-}, i_{+}, i_{0}\right) \in \mathrm{i}(\mathcal{A})$. Thus, in [2], the inertia set $S_{n}^{*}=\{(0, n, 0),(0, n-1,1),(1, n-1,0),(n, 0,0),(n-1,0,1),(n-1,1,0)\}$ was introduced.

An $n \times n$ zero-nonzero pattern (resp. sign pattern) $\mathcal{A}$ allows $\mathbb{S}_{n}^{*}\left(\right.$ resp. $\left.\mathbb{S}_{n}\right)$ if $\mathbb{S}_{n}^{*} \subseteq \mathrm{i}(\mathcal{A})\left(\right.$ resp. $\mathbb{S}_{n} \subseteq \mathrm{i}(\mathcal{A})$ ), and requires $\mathbb{S}_{n}^{*}\left(\right.$ resp. $\left.\mathbb{S}_{n}\right)$ if $\mathbb{S}_{n}^{*}=\mathrm{i}(\mathcal{A})\left(\right.$ resp. $\mathbb{S}_{n}=\mathrm{i}(\mathcal{A})$ ).

A related set of refined inertias $\mathbb{H}_{n}$ was introduced for sign patterns in [3]. The refined inertia of a square real matrix $B$, denoted by $\operatorname{ri}(B)$, is the ordered 4 -tuple $\left(n_{+}(B), n_{-}(B), n_{z}(B), 2 n_{p}(B)\right)$, where $n_{+}(B)$ (resp., $\left.n_{-}(B)\right)$ is the number of eigenvalues of $B$ with positive (resp., negative) real part, $n_{z}(B)$ is the number of

${ }^{*}$ Received by the editors on August 4, 2018. Accepted for publication on May 28, 2020. Handling Editor: Bryan L. Shader. Corresponding Author: Wei Gao.

${ }^{\dagger}$ Department of Mathematics, Pennsylvania State University at Abington, Abington, PA, 19001, USA (wvg5121@psu.edu).

¥Department of Mathematics, North University of China, Taiyuan, Shanxi 030051, P.R. China (ylshao@nuc.edu.cn). 
zero eigenvalues of $B$, and $2 n_{p}(B)$ is the number of pure imaginary (nonzero) eigenvalues of $B$. For $n \geq 2$, $\mathbb{H}_{n}=\{(0, n, 0,0),(0, n-2,0,2),(2, n-2,0,0)\}$. The set $\mathbb{H}_{n}$ can signal the onset of periodic solutions by a pair of nonzero pure imaginary eigenvalues in dynamical systems. For further results on $\mathbb{H}_{n}$, see $[4,5,6,8,9]$. In [1], the concept $\mathbb{H}_{n}$ for sign patterns is expanded to $\mathbb{H}_{n}^{*}$ for zero-nonzero patterns. For further results on $\mathbb{H}_{n}^{*}$, see [7].

A zero-nonzero pattern $\mathcal{A}$ is reducible if it is permutation similar to a pattern of the form

$$
\left[\begin{array}{cc}
\mathcal{A}_{11} & \mathcal{A}_{12} \\
0 & \mathcal{A}_{22}
\end{array}\right]
$$

where $\mathcal{A}_{11}, \mathcal{A}_{22}$ are square and non-vacuous. A zero-nonzero pattern is irreducible if it is not reducible.

There is a natural association between digraphs and zero-nonzero patterns. For an $n \times n$ zero-nonzero pattern $\mathcal{A}$, the associated digraph $D(\mathcal{A})$ has $n$ vertices $v_{1}, v_{2}, \ldots, v_{n}$, an arc from vertex $v_{i}$ to vertex $v_{j}$ if and only if the $(i, j)$-entry of $\mathcal{A}$ is nonzero, and a loop at vertex $v_{i}$ if and only if the $(i, i)$-entry of $\mathcal{A}$ is nonzero. Two digraphs are equivalent if and only if their associated zero-nonzero patterns are equivalent.

A simple cycle of length $l$ or an $l$-cycle in a digraph $D$ is a sequence of arcs of the form $C=v_{i_{1}} v_{i_{2}}, v_{i_{2}} v_{i_{3}}$, $\ldots, v_{i_{l}} v_{i_{1}}$, where $v_{i_{1}}, \ldots, v_{i_{l}}$ are distinct vertices. A composite cycle $C$ in a digraph $D$ is a vertex disjoint union of simple cycles, say $C=C_{1} \cup C_{2} \cup \cdots \cup C_{k}$. If the length of $C_{i}$ is $l_{i}$, then the length of $C$ is $\sum_{i=1}^{k} l_{i}$.

In [2], it is shown that a zero-nonzero pattern of order 2 allows $\mathbb{S}_{2}^{*}$ if and only if every entry in the zero-nonzero pattern is $*$. The authors also describe all irreducible nonequivalent zero-nonzero patterns of order 3 and 4 that allow $\mathbb{S}_{n}^{*}$. For zero-nonzero patterns requiring $\mathbb{S}_{n}^{*}$, it is proved that for $2 \leq n \leq 4$, there are no irreducible zero-nonzero patterns of order $n$ that require $\mathbb{S}_{n}^{*}$. For $n \geq 5$, this question is open.

In this paper, we study zero-nonzero patterns that allow or require $\mathbb{S}_{n}^{*}$. In Section 3 , we first prove that there are no irreducible zero-nonzero patterns of order $n \geq 5$ that require $\mathbb{S}_{n}^{*}$. So together with the result in [2], there are no irreducible zero-nonzero patterns of order $n \geq 2$ that require $\mathbb{S}_{n}^{*}$. Moreover, we can prove that there are no reducible zero-nonzero patterns of order $n \geq 2$ that require $\mathbb{S}_{n}^{*}$. In Section 4 , we give a complete characterization of zero-nonzero star patterns of order $n \geq 3$ that allow $\mathbb{S}_{n}^{*}$.

\section{Preliminaries.}

REMARK 2.1. Let $\mathcal{A}$ be a zero-nonzero pattern of order $n$. If $\mathcal{A}$ requires $\mathbb{S}_{n}^{*}$, then by the definition of $\mathbb{S}_{n}^{*}$, it is clear that for any $B \in Q(\mathcal{A})$, either $i_{+}(B) \leq 1$ or $i_{-}(B) \leq 1$.

LEMMA 2.2. Let $n \geq 4$ and $n \times n$ matrix

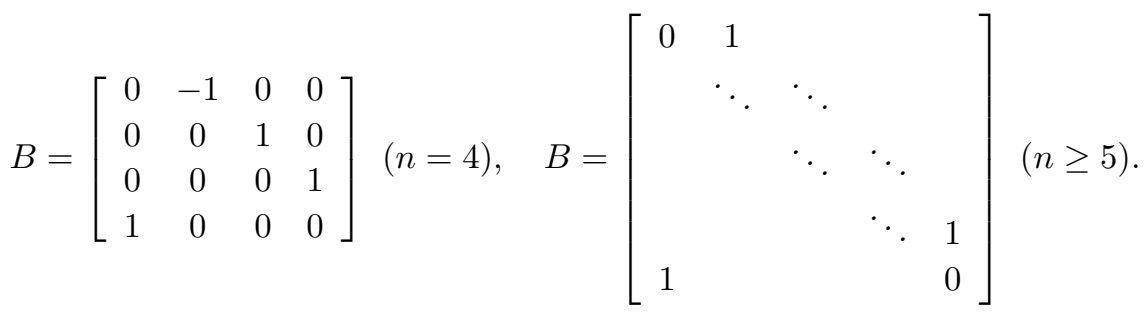

Then $i_{+}(B) \geq 2$ and $i_{-}(B) \geq 2$. 
Proof. When $n=4$, the characteristic polynomial of $B$ is

$$
\operatorname{det}(\lambda I-B)=\left|\begin{array}{cccc}
\lambda & 1 & 0 & 0 \\
0 & \lambda & -1 & 0 \\
0 & 0 & \lambda & -1 \\
-1 & 0 & 0 & \lambda
\end{array}\right|=\lambda^{4}+1=\left(\lambda^{2}+\sqrt{2} \lambda+1\right)\left(\lambda^{2}-\sqrt{2} \lambda+1\right) .
$$

So, $\mathrm{i}(B)=(2,2,0,0)$ and the lemma holds.

When $n \geq 5$, the characteristic polynomial of $B$ is

$$
\operatorname{det}(\lambda I-B)=\left|\begin{array}{ccccc}
\lambda & -1 & & & \\
& \ddots & \ddots & & \\
& & \ddots & \ddots & \\
& & & \ddots & -1 \\
-1 & & & & \lambda
\end{array}\right|=\lambda^{n}-1
$$

It is easy to see that $\sigma(B)=\left\{\cos \left(\frac{2 k \pi}{n}\right)+\mathrm{i} \sin \left(\frac{2 k \pi}{n}\right) \mid k=1,2, \ldots, n\right\}$, and so $i_{+}(B) \geq 2$ and $i_{-}(B) \geq 2$. The lemma holds.

A zero-nonzero pattern $\mathcal{B}$ is a subpattern of a zero-nonzero pattern $\mathcal{A}$ if $\mathcal{B}$ is obtained from $\mathcal{A}$ by replacing some (or possibly none) of the nonzero entries of $\mathcal{A}$ with zeros. $\mathcal{A}$ is a superpattern of $\mathcal{B}$ if $\mathcal{B}$ is a subpattern of $\mathcal{A}$.

Lemma 2.3. Let $\mathcal{A}$ be a zero-nonzero pattern of order $n$ and $\mathcal{A}_{1}$ be a subpattern of $\mathcal{A}$. If there exists $B_{0} \in Q\left(\mathcal{A}_{1}\right)$ such that $i_{+}\left(B_{0}\right) \geq 2$ and $i_{-}\left(B_{0}\right) \geq 2$, then $\mathcal{A}$ does not require $\mathbb{S}_{n}^{*}$.

Proof. Note that the eigenvalues of a matrix can be arranged so that they are continuous functions of the entries ([10]). If the condition holds, then there exists a matrix $B \in Q(\mathcal{A})$ such that $i_{+}(B) \geq 2$ and $i_{-}(B) \geq 2$. So $\mathcal{A}$ does not require $\mathbb{S}_{n}^{*}$ by Remark 2.1 .

LEmma 2.4. Let $\mathcal{A}$ be a zero-nonzero pattern of order $n$ and assume that $\mathcal{A}$ has $k$ nonzero diagonal entries. If $\mathcal{A}$ requires $\mathbb{S}_{n}^{*}$, then $1 \leq k \leq 3$.

Proof. First suppose $k=0$. Since the trace of $\mathcal{A}$ is zero, it is impossible to find $B \in Q(\mathcal{A})$ with $\mathrm{i}(B)=(0, n-1,1)$. This contradicts to $\mathcal{A}$ requiring $\mathbb{S}_{n}^{*}$.

Now suppose $k>4$. Without loss of generality, assume that the nonzero diagonal entries of $\mathcal{A}$ are $a_{11}, a_{22}, \ldots, a_{k k}$. By emphasizing $a_{11}, a_{22}, a_{33}, a_{44}$ and taking $a_{11}>0, a_{22}>0, a_{33}<0, a_{44}<0$, we can get a matrix $B \in Q(\mathcal{A})$ such that $i_{+}(B) \geq 2$ and $i_{-}(B) \geq 2$. By Remark 2.1, $\mathcal{A}$ does not require $\mathbb{S}_{n}^{*}$. Thus, the result follows.

Lemma 2.5. Let $\mathcal{A}$ be a zero-nonzero pattern of order $n$. If $D(\mathcal{A})$ contains a composite cycle that consists of one 2 -cycle and two loops, then $\mathcal{A}$ does not require $\mathbb{S}_{n}^{*}$.

Proof. Without loss of generality, assume that the 2-cycle is $v_{1} v_{2} v_{1}$ and two loops are on $v_{3}$ and $v_{4}$. Take an $n \times n$ matrix $B_{0}=\left(b_{i j}\right)$ with $b_{12}=b_{21}=b_{33}=1, b_{44}=-1$, and other entries equal to zero. It is clear that $i_{+}\left(B_{0}\right)=2$ and $i_{-}\left(B_{0}\right)=2$. Thus, $\mathcal{A}$ does not require $\mathbb{S}_{n}^{*}$ by Lemma 2.3.

A zero-nonzero pattern $\mathcal{A}$ is combinatorially singular if $B$ is singular for all $B \in Q(\mathcal{A})$, and $\mathcal{A}$ is combinatorially nonsingular if $B$ is nonsingular for all $B \in Q(\mathcal{A})$. 
Lemma 2.6. ([2]) If an $n \times n$ zero-nonzero pattern $\mathcal{A}$ requires or allows $\mathbb{S}_{n}^{*}$, then $\mathcal{A}$ is not combinatorially singular, and not combinatorially nonsingular.

Lemma 2.7. If an $n \times n$ zero-nonzero pattern $\mathcal{A}$ allows $\mathbb{S}_{n}$, then $\mathcal{A}$ allows $\mathbb{S}_{n}^{*}$. And if an $n \times n$ sign pattern $\mathcal{A}$ allows or requires $\mathbb{S}_{n}$, then the associated zero-nonzero pattern allows $\mathbb{S}_{n}^{*}$.

Proof. Note that for zero-nonzero pattern $\mathcal{A}$, the inertia $\left(i_{+}, i_{-}, i_{0}\right) \in i(\mathcal{A})$ if and only if its reversal $\left(i_{-}, i_{+}, i_{0}\right) \in i(\mathcal{A})$.

\section{Zero-nonzero patterns of order $n$ do not require $\mathbb{S}_{n}^{*}$.}

Lemma 3.1. Let $\mathcal{A}$ be a zero-nonzero pattern of order $n \geq 5$. If $D(\mathcal{A})$ satisfies one of the following two conditions, then $\mathcal{A}$ does not require $\mathbb{S}_{n}^{*}$.

(1) $D(\mathcal{A})$ contains a s-cycle with $s \geq 4$;

(2) $D(\mathcal{A})$ contains a $k$-cycle and a $t$-cycle that are vertex disjoint with $1 \leq k, t \leq 3$ and $k+t \geq 4$.

Proof. Denote $V(D(\mathcal{A}))=\left\{v_{1}, v_{2}, \ldots, v_{n}\right\}$. Consider the following two cases.

Case 1. $D(\mathcal{A})$ contains a $s$-cycle $C_{s}$ with $s \geq 4$.

Without loss of generality, assume that $C_{s}=v_{1} v_{2} \cdots v_{s} v_{1}$. If $s=4$, then take an $n \times n$ matrix $B_{0}=\left(b_{i j}\right)$ with $b_{12}=-1, b_{23}=\cdots=b_{s-1, s}=b_{s, 1}=1$, and the other entries of $B_{0}$ equal to zero. If $s>4$, then take an $n \times n$ matrix $B_{0}=\left(b_{i j}\right)$ with $b_{12}=b_{23}=\cdots=b_{s-1, s}=b_{s, 1}=1$, and the other entries of $B_{0}$ equal to zero. By Lemma $2.2, i_{+}\left(B_{0}\right) \geq 2$ and $i_{-}\left(B_{0}\right) \geq 2$. Thus, $\mathcal{A}$ does not require $\mathbb{S}_{n}^{*}$ by Lemma 2.3 .

Case 2. $D(\mathcal{A})$ contains a $k$-cycle $C_{k}$ and a $t$-cycle $C_{t}$ that are vertex disjoint with $1 \leq k, t \leq 3$ and $k+t \geq 4$.

Without loss of generality, assume that $C_{k}=v_{1} v_{2} \cdots v_{k} v_{1}$ and $C_{t}=v_{k+1} v_{k+2} \cdots v_{k+t} v_{k+1}$. Up to equivalence, there are the following four cases.

- $k=3$ and $t=1$;

- $k=2$ and $t=2$;

- $k=2$ and $t=3$;

- $k=3$ and $t=3$.

If $k=3$ and $t=1$, take an $n \times n$ matrix $B_{0}=\left(b_{i j}\right)$ with $b_{12}=b_{23}=b_{31}=1, b_{44}=1$ and the other entries equal to zero. Then $i_{+}\left(B_{0}\right)=2$ and $i_{-}\left(B_{0}\right)=2$, and $\mathcal{A}$ does not require $\mathbb{S}_{n}^{*}$ by Lemma 2.3.

If $k=2$ and $t=2$, take an $n \times n$ matrix $B_{0}=\left(b_{i j}\right)$ with $b_{12}=b_{21}=b_{34}=b_{43}=1$, and the other entries equal to zero. Then $i_{+}\left(B_{0}\right)=2$ and $i_{-}\left(B_{0}\right)=2$, and $\mathcal{A}$ does not require $\mathbb{S}_{n}^{*}$ by Lemma 2.3.

If $k=2$ and $t=3$, take an $n \times n$ matrix $B_{0}=\left(b_{i j}\right)$ with $b_{12}=b_{21}=b_{34}=b_{45}=b_{53}=1$, and the other entries equal to zero. Then $i_{+}\left(B_{0}\right)=2$ and $i_{-}\left(B_{0}\right)=3$, and $\mathcal{A}$ does not require $\mathbb{S}_{n}^{*}$ by Lemma 2.3.

If $k=3$ and $t=3$, take an $n \times n$ matrix $B_{0}=\left(b_{i j}\right)$ with $b_{12}=b_{23}=b_{31}=1, b_{45}=b_{56}=b_{64}=-1$, and the other entries equal to zero. Then $i_{+}\left(B_{0}\right)=3$ and $i_{-}\left(B_{0}\right)=3$, and $\mathcal{A}$ does not require $\mathbb{S}_{n}^{*}$ by Lemma 2.3 .

TheOREM 3.2. There are no irreducible zero-nonzero patterns of order $n \geq 2$ that require $\mathbb{S}_{n}^{*}$.

Proof. Based on the result in [2], we only need to prove the result when $n \geq 5$. 
Electronic Journal of Linear Algebra, ISSN 1081-3810

A publication of the International Linear Algebra Society

Volume 36, pp. 503-510, July 2020.

Let $D(\mathcal{A})$ be the associated digraph of $\mathcal{A}$ with vertex set $\left\{v_{1}, v_{2}, \ldots, v_{n}\right\}$. By Lemma 2.6, we may assume that $\mathcal{A}$ is not combinatorially singular. Thus, there exists a composite cycle $C=C_{1} \cup C_{2} \cup \cdots \cup C_{k}$ of length $n$ in $D(\mathcal{A})$. Denote the lengths of $C_{1}, C_{2}, \ldots, C_{k}$ by $l_{1}, \ldots, l_{k}$, respectively. Without loss of generality, assume that $l_{1} \leq \cdots \leq l_{k}$.

Consider the following four cases.

Case 1. $l_{k} \geq 4$. Then $\mathcal{A}$ does not require $\mathbb{S}_{n}^{*}$ by Lemma 3.1.

Case 2. $l_{k}=3$. Note that $k \geq 2$ since $n \geq 5$. Then $\mathcal{A}$ does not require $\mathbb{S}_{n}^{*}$ by Lemma 3.1.

Case 3. $l_{k}=2$. If $l_{k-1}=2$, then $D(\mathcal{A})$ contains two vertex disjoint 2-cycles that do not have common vertices. By Lemma 3.1, $\mathcal{A}$ does not require $\mathbb{S}_{n}^{*}$. Otherwise, since $n \geq 5$, it is clear that $D(\mathcal{A})$ contains a composite cycle that consists of one 2-cycle and two loops. By Lemma 2.5, $\mathcal{A}$ does not require $\mathbb{S}_{n}^{*}$.

Case $4 . l_{k}=1$. Since $n \geq 5, \mathcal{A}$ has at least five nonzero diagonal vertices. By Lemma 2.4, $\mathcal{A}$ does not require $\mathbb{S}_{n}^{*}$.

THEOREM 3.3. There are no reducible zero-nonzero patterns of order $n \geq 2$ that require $\mathbb{S}_{n}^{*}$.

Proof. Let $\mathcal{A}$ be a reducible zero-nonzero pattern of order $n$. Then there is a permutation zero-nonzero pattern $\mathcal{P}$ such that

$$
\mathcal{P}^{T} \mathcal{A} \mathcal{P}=\left[\begin{array}{cc}
\mathcal{A}_{m} & \# \\
0 & \mathcal{A}_{n-m}
\end{array}\right],
$$

where $\#$ is an $m \times(n-m)$ zero-nonzero pattern and $1 \leq m \leq n-1$.

Suppose that $\mathcal{A}$ requires $\mathbb{S}_{n}^{*}$. Then $\{(0, n, 0),(n, 0,0)\} \subseteq \mathrm{i}(\mathcal{A})$, and so $\{(0, m, 0),(m, 0,0)\} \subseteq \mathrm{i}\left(\mathcal{A}_{m}\right)$ and $\{(0, n-m, 0),(n-m, 0,0)\} \subseteq \mathrm{i}\left(\mathcal{A}_{n-m}\right)$. Thus, $\{(m, n-m, 0),(n-m, m, 0)\} \subseteq \mathrm{i}(\mathcal{A})$. So $m=1$ or $n-m=1$.

If $m=1$ and $n-m=1$, then $n=2$, and $\mathcal{A}_{m}=\mathcal{A}_{n-m}=(*)$. Thus, $\mathrm{i}(\mathcal{A})=\{(0,2,0),(1,1,0),(2,0,0)\}$, and so $\mathcal{A}$ does not require $\mathbb{S}_{n}^{*}$.

If only one of $m$ and $n-m$ is equal to one, then $n \geq 3$. Without loss of generality, assume $m=1$, that is, $\mathcal{A}_{m}=(*)$. Then $\mathrm{i}\left(\mathcal{A}_{m}\right)=\{(1,0,0),(0,1,0)\}$. Since $\mathcal{A}$ requires $\mathbb{S}_{n}^{*}$, we must have $\{(0, n-2,1),(n-2,0,1)\} \subseteq$ $\mathrm{i}\left(\mathcal{A}_{n-m}\right)$. Thus, $\{(1, n-2,1),(n-2,1,1)\} \subseteq \mathrm{i}(\mathcal{A})$, a contradiction.

Theorems 3.2 and 3.3 give the main result of this section as follows.

THEOREM 3.4. There are no zero-nonzero patterns of order $n \geq 2$ that require $\mathbb{S}_{n}^{*}$.

4. Zero-nonzero star patterns that allow $\mathbb{S}_{n}^{*}$. Up to equivalence, an $n \times n$ zero-nonzero star pattern can be represented in the following form

$$
\mathcal{A}=\left[\begin{array}{cccc}
a_{11} & * & \cdots & * \\
* & a_{22} & & \\
\vdots & & \ddots & \\
* & & & a_{n n}
\end{array}\right]
$$

where $a_{i i} \in\{*, 0\}$ for $i=1,2, \ldots, n$.

In [2], it is shown that a zero-nonzero pattern of order 2 allows $\mathbb{S}_{2}^{*}$ if and only if every entry in the zero-nonzero pattern is $*$. In this section, we give a complete characterization of zero-nonzero star patterns 
Electronic Journal of Linear Algebra, ISSN 1081-3810

of order $n \geq 3$ that allow $\mathbb{S}_{n}^{*}$.

TheOREm 4.1. Let $n \geq 3$ and $\mathcal{A}$ be a zero-nonzero star pattern of order $n$. Then $\mathcal{A}$ allows $\mathbb{S}_{n}^{*}$ if and only if $\mathcal{A}$ is equivalent to one of the following patterns

$$
\mathcal{A}_{1}=\left[\begin{array}{ccccc}
* & * & \ldots & \ldots & * \\
* & * & & & \\
\vdots & & * & & \\
\vdots & & & \ddots & \\
* & & & & *
\end{array}\right] \text { and } \mathcal{A}_{2}=\left[\begin{array}{ccccc}
0 & * & \cdots & \cdots & * \\
* & * & & & \\
\vdots & & * & & \\
\vdots & & & \ddots & \\
* & & & *
\end{array}\right]
$$

When $\mathcal{A}$ allows $\mathbb{S}_{n}^{*}, \mathcal{A}$ has a signing that allows $\mathbb{S}_{n}$

Proof. Let $\mathcal{A}$ be a zero-nonzero star pattern of order $n$. Up to equivalence, we may assume that $\mathcal{A}$ is in the form (4.1).

Necessity. If at least two of $a_{22}, \ldots, a_{n n}$ are equal to zero, then $\mathcal{A}$ is combinatorially singular, and $\mathcal{A}$ doesn't allow $\mathbb{S}_{n}^{*}$ by Lemma 2.6. If exactly one of $a_{22}, \ldots, a_{n n}$ is equal to zero, then $\mathcal{A}$ is combinatorially nonsingular, and $\mathcal{A}$ doesn't allow $\mathbb{S}_{n}^{*}$ by Lemma 2.6. Thus, up to equivalence, we may assume $a_{i i}=*$ for $i=2,3, \ldots, n$, that is, $\mathcal{A}$ is equivalent to one of patterns $\mathcal{A}_{1}$ and $\mathcal{A}_{2}$.

For sufficiency, we consider the following two cases.

Case 1. $\mathcal{A}$ is equivalent to $\mathcal{A}_{1}$.

Without loss of generality, assume that $\mathcal{A}=\mathcal{A}_{1}$. Take

$$
B=\left[\begin{array}{ccccc}
a & 1 & 1 & \cdots & 1 \\
b & -1 & & & \\
-1 & & -1 & & \\
\vdots & & & \ddots & \\
-1 & & & & -1
\end{array}\right] \in Q(\mathcal{A})
$$

where $a \neq 0$ and $b \neq 0$. Then the characteristic polynomial of $B$ is

$$
f_{B}(x)=|x I-B|=\left|\begin{array}{ccccc}
x-a & -1 & -1 & \cdots & -1 \\
-b & x+1 & & & \\
1 & & x+1 & & \\
\vdots & & & \ddots & \\
1 & & & & x+1
\end{array}\right|
$$


Electronic Journal of Linear Algebra, ISSN 1081-3810

A publication of the International Linear Algebra Society

Volume 36, pp. 503-510, July 2020.

By subtracting the second column from the $k$ th column, $k=3,4, \ldots, n$, respectively, we have

$$
\begin{aligned}
& f_{B}(x)=\left|\begin{array}{ccccc}
x-a & -1 & 0 & \cdots & 0 \\
-b & x+1 & -(x+1) & \cdots & -(x+1) \\
1 & & x+1 & & \\
\vdots & & & \ddots & \\
1 & & & & x+1
\end{array}\right| \\
& =(x-a)(x+1)^{n-1}+\left|\begin{array}{cccc}
-b & -(x+1) & \cdots & -(x+1) \\
1 & x+1 & & \\
\vdots & & \ddots & \\
1 & & & x+1
\end{array}\right|_{n-1} \\
& =(x-a)(x+1)^{n-1}+\left|\begin{array}{cccc}
n-b-2 & 0 & \cdots & 0 \\
1 & x+1 & & \\
\vdots & & \ddots & \\
1 & & & x+1
\end{array}\right|_{n-1} \\
& =(x-a)(x+1)^{n-1}+(n-b-2)(x+1)^{n-2} \\
& =(x+1)^{n-2}\left(x^{2}+(1-a) x+n-a-b-2\right) \text {. }
\end{aligned}
$$

(1) If $a=\frac{1}{3}$ and $b=n-\frac{5}{2}$, then $f_{B}(x)=(x+1)^{n-2}\left(x^{2}+\frac{2}{3} x+\frac{1}{6}\right)$, and so $\mathrm{i}(B)=(0, n, 0)$.

(2) If $a=\frac{1}{2}$ and $b=n-\frac{5}{2}$, then $f_{B}(x)=(x+1)^{n-2}\left(x^{2}+\frac{1}{2} x\right)$, and so $\mathrm{i}(B)=(0, n-1,1)$.

(3) If $a=1$ and $b=n-2$, then $f_{B}(x)=(x+1)^{n-1}(x-1)$, and so $\mathrm{i}(B)=(1, n-1,0)$. Thus, $\operatorname{sgn}(B)$ allows $\mathbb{S}_{n}$. By Lemma $2.7, \mathcal{A}$ allows $\mathbb{S}_{n}^{*}$, and $\mathcal{A}$ has a signing that allows $\mathbb{S}_{n}$.

Case 2. $\mathcal{A}$ is equivalent to $\mathcal{A}_{2}$.

Without loss of generality, assume that $\mathcal{A}=\mathcal{A}_{2}$. Take

$$
C=\left[\begin{array}{ccccc}
0 & 1 & 1 & \cdots & 1 \\
c & -1 & & & \\
-1 & & -1 & & \\
\vdots & & & \ddots & \\
-1 & & & & -1
\end{array}\right] \in Q(\mathcal{A})
$$

where $c \neq 0$. By the similar steps as in Case 1 , we can get the characteristic polynomial of $C$

$$
f_{C}(x)=|x I-C|=(x+1)^{n-2}\left(x^{2}+x+n-c-2\right) .
$$

(1) If $c=\frac{1}{2}$, then $f_{C}(x)=(x+1)^{n-2}\left(x^{2}+x+n-\frac{5}{2}\right)$, and so $\mathrm{i}(C)=(0, n, 0)$.

(2) If $c=n-2$, then $f_{C}(x)=x(x+1)^{n-1}$, and so $\mathrm{i}(C)=(0, n-1,1)$.

(3) If $c=n$, then $f_{C}(x)=(x+1)^{n-2}(x+2)(x-1)$, and so $\mathrm{i}(C)=(1, n-1,0)$. Thus, $\operatorname{sgn}(C)$ allows $\mathbb{S}_{n}$. By Lemma $2.7, \mathcal{A}$ allows $\mathbb{S}_{n}^{*}$, and $\mathcal{A}$ has a signing that allows $\mathbb{S}_{n}$. 
Acknowledgements. The authors would like to thank the anonymous referee for valuable suggestions which greatly improved the exposition of the paper.

\section{REFERENCES}

[1] A.H. Berliner, D.D. Olesky, and P. van den Driessche. Sets of refined inertias of zero-nonzero patterns. Linear Algebra and its Applications, 516:243-263, 2017.

[2] A.H. Berliner, D.D. Olesky, and P. van den Driessche. Inertia sets allowed by matrix patterns. Electronic Journal of Linear Algebra, 34:343-355, 2018.

[3] E. Bodine, L. Deaett, J.J. McDonald, D.D. Olesky, and P. van den Driessche. Sign patterns that require or allow particular refined inertias. Linear Algebra and its Applications, 437(9):2228-2242, 2012.

[4] W. Gao, Z. Li, and L. Zhang. Sign patterns that require $\mathbb{H}_{n}$ exist for each $n \geq 4$. Linear Algebra and its Applications, 489:15-23, 2016.

[5] W. Gao, Z. Li, and L. Zhang. Characterization of star sign patterns that require $\mathbb{H}_{n}$. Linear Algebra and its Applications, 499:43-65, 2016.

[6] W. Gao and Z. Li. Path sign patterns of order $n \geq 5$ do not require $\mathbb{H}_{n}$. Linear Algebra and its Applications, 532:99-126, 2017.

[7] W. Gao. Zero-nonzero patterns of order $n \geq 4$ do not require $\mathbb{H}_{n}^{*}$. Linear Algebra and its Applications, 554:1-14, 2018.

[8] C. Garnett, D.D. Olesky, and P. van den Driessche. Refined inertias of tree sign patterns. Electronic Journal of Linear Algebra, 26:620-635, 2013.

[9] C. Garnett, D.D. Olesky, and P. van den Driessche. A note on sign patterns of order 3 that require particular refined inertias. Linear Algebra and its Applications, 450:293-300, 2014.

[10] R.A. Horn and C.R. Johnson. Matrix Analysis. Cambridge University Press, Cambridge, 1985.

[11] I.-J. Kim, J.J. McDonald, D.D. Olesky, and P. van den Driessche. Inertias of zero-nonzero patterns. Linear and Multilinear Algebra, 55(3):229-238, 2007. 